\title{
ZNF217 is Overexpressed and Enhances Cell Migration and Invasion in Colorectal Carcinoma
}

\author{
Zi-Chao Zhang ${ }^{1}$, Li-Qiang Zheng ${ }^{2}$, Li-Jie Pan ${ }^{1}$, Jin-Xing Guo ${ }^{1}$, Guo-Shan Yang ${ }^{1 *}$
}

\begin{abstract}
Background: To investigate the expression and clinical significance of zinc finger protein 217 (ZNF217) in human colorectal carcinoma (CRC). Materials and Methods: The expression of ZNF217 in 60 CRC tissues and matched tumor adjacent tissues, collected between January 2013 and June 2014, was assessed immunohistochemically. The relationship between the expression of ZNF217 and clinicopathlogical features was analyzed by Pearson chi-square test. In addition, siRNA was used to down-regulate the expression of ZNF217 in CRC cells. The effects of ZNF217 for cell migration and invasion were measured by wound healing assay and transwell assay, respectively. Results: The expression level of ZNF217 was significantly higher in CRC tissues than in tumor adjacent tissues $(p<\mathbf{0 . 0 5})$, positively correlating with tumor size, lymphatic metastasis and advanced TNM stage $(p<0.05)$. Down-regulation of ZNF217 in CRC cells could significantly suppress cell migration and invasion. Conclusions: ZNF217 is overexpressed in colorectal carcinoma tissues and is associated with tumor malignant clinicopathological features. ZNF217 may promote CRC progression by inducing cell migration and invasion.
\end{abstract}

Keywords: ZNF217 - expression - colorectal carcinoma - migration - invasion

Asian Pac J Cancer Prev, 16 (6), 2459-2463

\section{Introduction}

Colorectal carcinoma (CRC) is one of the most common digestive cancers in human (Mahmoud et al., 2014). Metastases, especially lymphatic metastasis and liver metastasis, are the main risk factors to restrict the survival of CRC patients. Recently, the monoclonal antibodies against EGFR and VEGF, such as cetuximab, panitumumab and bevacizumab, are in clinical development and have shown clinical benefit in metastatic CRC patients (Zhang et al., 2014; Han et al., 2014). The investigation of effective biomarkers and therapeutic molecular targets has been a significant hotspot of CRC throughout the world.

Zinc finger protein 217 (ZNF217) is located in the human chromosomes of 20q13.2 (Rahman et al., 2012) and has been demonstrated as a crucial oncogene in numerous human cancers. As a role of nuclear transcriptional regulatory factor, ZNF217 obtains a special located signal and combines with DNA to impede the transcription of some genes, such as TGF- $\beta$ (Thillainadesan et al., 2012) and P53 (Thillainadesan et al., 2012). In breast cancer, ZNF217 had been demonstrated significantly overexpressed and associated with tumor growth(Frietze, et al., 2014) and metastases (Vendrell et al., 2012), Kaplan-Meier survival analysis revealed that eptopic expression of ZNF217 indicated a worse OS and DFS time after operation (Letessier et al., 2006). Moreover, the tumor-promoting action of ZNF217 was also reported in pancreatic cancer (Letessier et al., 2006), oesophageal adenocarcinoma (Geppert et al., 2014) and malignant glioma (Mao et al., 2011). However, the expression and functions of ZNF217 in human colorectal carcinoma were still absent of study.

In this study, we demonstrated that the expression of ZNF217 was overexpressed in CRC tissues and associated with tumor malignant clinicopathologic features. Downregulated the expression of ZNF217 could impair the ability of cell migration and invasion in vitro. Our results suggest that ZNF217 may promote tumor progression by promoting cell metastasis in CRC.

\section{Materials and Methods}

\section{Ethical review}

All protocols were approved by the First Affiliated Hospital of Tsinghua University Ethics Committee according to the 1975 Declaration of Helsinki. Informed consent was obtained and signed by each patient.

\section{Patients and samples}

A total of 60 patients including 32 males and 28 females were enrolled in our study from January 2013 to June 2014. The range of age was from 34 to 68 year, and 


\section{Zi-chao ZHANG et al}

the average age was 49 year. All patients didn't accept chemotherapy and radiotherapy before surgery. The CRC tissues and tumor adjacent tissues $(>2 \mathrm{~cm}$ distance of the surgical boundary) were stored in $4 \%$ paraformaldehyde solution for immunohistochemistry (IHC) or liquid nitrogen for qRT-PCR and western blot.

\section{RNA isolation and $q R T-P C R$}

Total RNA was isolated from CRC tissues and CRC cells using TRIZOL ${ }^{\circledR}$ reagent (Invitrogen, USA) according to its manufacturer. The value of OD260/280 for RNA located in 1.9 2.1 was qualified. cDNA was synthetized using the RevertidTM First Strand cDNA Synthesis Kit (Fermentas, USA) as the follow system: $70^{\circ} \mathrm{C}$ for $5 \mathrm{~min}$, $37^{\circ} \mathrm{C}$ for $1 \mathrm{~h}$ and $85^{\circ} \mathrm{C}$ for $5 \mathrm{~min} .2 \mu \mathrm{L}$ cNDA was served as a template to be amplified by Real-time PCR using SYBR ${ }^{\circ}$ Premix Ex TaqTM II (Tli RNaseH Plus, Takara, Japan) using the conditions as the manufacture instructed. The human $\beta$-actin gene was served as a control gene. The primers were synthesized by ShengGong Co, Ltd and the sequences were displayed in Table1. Relative mRNA expression was calculated with the $2^{-\Delta \Delta \mathrm{CT}}$ method. Every sample was duplicate detected for three times.

\section{Immumohistochemical staining}

Immunohistochemistry was performed on paraformaldehyde-fixed paraffin sections. Sections were processed with xylene, alcohol and PBS, sequentially. Tissues were incubated with ZNF217 (sc-67223, Santa Cruz, USA) (1:100) antibodies at $4^{\circ} \mathrm{C}$ overnight. Biotinylated secondary antibodies were used to combine ZNF217 antibodies, detected by HRP-streptavidin conjugates and visualized by DAB. The staining results for the ZNF217 protein was semi-quantitatively calculated by multiplying the staining intensity and the percentage of positive normal cells as the reference (Li et al., 2014) reported.

\section{Cell culture}

The human normal colorectal cell line FHC and four CRC cell lines HCT116, HT29, SW480, SW620 were obtained from the Institute of Biochemistry and Cell Biology, Chinese Academy of Sciences (Shanghai, China). Cells were maintained under recommended culture conditions and cultured in a $37^{\circ} \mathrm{C}$ humidified incubator with $5 \% \mathrm{CO}_{2}$. The logarithmic growth phase cells were harvested for further assays.

\section{Protein isolation and western blot}

Cells were washed with PBS for twice and cleaved with RIPA reagent on the ice. Lysate was centrifuge at $15000 \mathrm{r} / \mathrm{min}$ for $15 \mathrm{~min}$. The supernate was obtained and detected the protein content via BCA Kit (Milipore, USA). Proteins were separated by vertical electrophoresis and transfered to PVDF membrane (Milipore, USA). ZNF217 (1:1000) and $\beta$-actin (sc-4778, Santa Cruz, USA) (1:5000) antibodies were used to detected the expression of ZNF217 and $\beta$-actin, respectively. Secondary HRP-conjugated goat anti-rabbit or anti-mouse antibody (Abgent, USA) were used at a 1:5000 dilution and developed by the ECL Regent (Millipore, USA).

\section{siRNA transfection}

Both the mRNA and protein levels of ZNF217 in FHC, HCT116, HT29, SW480, SW620 cells were measured by qRT-PCR and western blot. The highest ZNF217 expression cell line was selected to transfect the specific siRNA against ZNF217 (sc-63249, Santa Cruz, USA) or scrambled siRNA (sc-37007, Santa Cruz, USA). Cells were seeded in 6-well plates at the concentration of $2 \times 10^{5} /$ well, and divided into two groups (ZNF217 siRNA group and scrambled siRNA group). $5 \mu \mathrm{L}$ Lipofectamine 2000 (Invitrogen, USA) was used as an to transfect the siRNA into cells according to the manufacturer. Cells were cultured in a $37^{\circ} \mathrm{C}$ humidified incubator with $5 \%$ $\mathrm{CO}_{2}$. Reduced serum medium was changed into complete medium after $6 \mathrm{~h}$ transfection. Cells were harvest after $48 \mathrm{~h}$ transfection, and then used for further experiments.

\section{Wound healing assay}

Cells were seeded in 6-well plates at high density and allowed to form cell monolayer overnight. A $200 \mu \mathrm{L}$ sterile plastic tip was used to create a wound line across the surface of plates, then scouring off the suspension cells with PBS. Cells were cultured in reduced serum medium in a $37^{\circ} \mathrm{C}$ humidified incubator with $5 \% \mathrm{CO}_{2}$ for $4 \mathrm{~h}$. The experiments were performed in triplicate.

\section{Cell invasion assay}

The $8 \mu \mathrm{m}$ pore-sized transwell inserts (Nalge Nunc, IL) were coated with matrigel (BD Biosciences, USA) at $1 \mathrm{mg} / \mathrm{ml}$ on the inner layer. ZNF217 siRNA or scrambled siRNA transfected CRC cells were resuspended with reduced serum DMEM medium and adjusted the density to $2.5 \times 10^{5} / \mathrm{ml}$. $200 \mu \mathrm{L}$ cell suspension was added into upper-well, and $750 \mu \mathrm{L}$ DMEM medium with $10 \%$ FBS was added into the lower-well. After completion, cells were incubated in a $37^{\circ} \mathrm{C}$ humidified incubator with $5 \%$ $\mathrm{CO}_{2}$ for 24 hours. Membranes were removed, wiped on the side facing the upper well, and stained with crystal violet. At least 6 representative images of each well were taken and cell numbers were counted using ImageJ software. The experiments were performed in triplicate.

\section{Statistical analysis}

Measurement date are presented as (Mean $\pm \mathrm{SD}$ ). The SPSS version 13.0 (SPSS, Chicago, USA) was used for Pearson chi-square test, two-tailed Student-t test and ANOVA. $p<0.05$ was considered statistically significant.

\section{Results}

\section{The eptopic expression of ZNF217 in CRC tissues}

qRT-PCR results revealed that, the relative mRNA expression of ZNF217 in CRC tissues was (5.093 \pm 0.359$)$, while that in tumor adjacent tissues was $(1.875 \pm 0.133)$. Two-tailed student-t analysis demonstrated that the expression of ZNF217 mRNA was significantly upregulated in CRC tissues than in tumor adjacent tissues $(\mathrm{t}=8.421, p<0.001$, Figure 1A). Furthermore, we detected the protein of ZN217 in both tumor and non-tumor tissues. As shown in figure $1 \mathrm{~B}$, the positive expression of ZNF217 in CRC tissues was $28.33 \%$ (17/60), 
by contrast, only $28.33 \%$ (17/60) of tumor adjacent tissues could be detected the positive expression. Pearson chisquare test convinced that the expression of ZNF217 protein in tumor and non-tumor tissues had a statistical difference $\left(\chi^{2}=34.478, p<0.001\right.$, Figure 1C, Table2).

\section{Clinical significance of ZNF217 expression in CRC}

In order to notarize the clinical significance of ZNF217 expression. We analyzed the relationship between ZNF217 expression and the clinicopathlogical features of CRC patients. The statistical data demonstrated that the expression of ZNF217 was correlated with larger tumor volume $(\geq 5 \mathrm{~cm})$, lymphatic metastasis and advanced TNM stage $(p<0.05)$. We may infer that high expression of ZNF217 in CRC tissues indicated a worse survival.

\section{Expression of ZNF217 in mCRC and nmCRC tissues}

In this enrolled group, 42 patients were diagnosed metastatic CRC, who existed lymphatic and (or) distant metastases. We detected the mRNA and protein of ZNF217 in metastatic CRC (mCRC) and non-metastatic CRC(nmCRC) tissues. Compared to nmCRC tissues, both mRNA (Figure 2A) and protein (Figure 2B\&2C) expression of ZNF217 was extremely overexpressed in mCRC tissues $(p<0.05)$.

siRNA down-regulated ZNF217 expression in SW620 cells

We detected the expression of ZNF217 in four CRC

Table 1. Real-time PCR Primer Sequences

\begin{tabular}{lll}
\hline Gene & \multicolumn{2}{c}{ primer sequences } \\
\hline ZNF217 Sense & 5'-GAGAAGCGAATGGTGAAAGC-3' \\
& Anti-sense & 5'-CAGCGCTCAAGTATGCAAAA-3' \\
$\beta$-actin & Sense & 5'-CTCCATCCTGGCCTCGCTGT-3' \\
& Anti-sense & 5'-GCTGTCACCTTCACCGTTCC-3' \\
\hline
\end{tabular}

Table 2. The Expression of ZNF217 in CRC and Tumor Adjacent Tissues $(n=60)$

\begin{tabular}{lcccc}
\hline & $\begin{array}{l}\text { ZNF217 } \\
\text { Positive }\end{array}$ & $\begin{array}{c}\text { ZNF217 } \\
\text { Negative }\end{array}$ & $\chi^{2}$ & $p$ \\
\hline CRC tissues & 49 & 11 & 34.478 & $<0.001^{*}$ \\
Tumor adjacent & 17 & 43 & & \\
tissues & & & & \\
$* p<0.05$ & & & &
\end{tabular}

cell lines HCT116, HT29, SW480, SW620 and normal colorectal cell line FHC. We found the expression of ZNF217 in CRC cells, especially SW620 cells were marked up-regulated than in FHC cells $(p<0.05$, Figure $3 \mathrm{~A} \& 3 \mathrm{~B})$. Consequently, we transfected the siRNA into SW620 cells to specifically down-regulate the expression of ZNF217. qRT-PCR and western blot were applied to confirm the effect of interference(Figure 3C\&3D).

Down-regulation of ZNF217 inhibits cell migration and invasion in SW620 cells

To determine whether eptopic expression of ZNF217
A

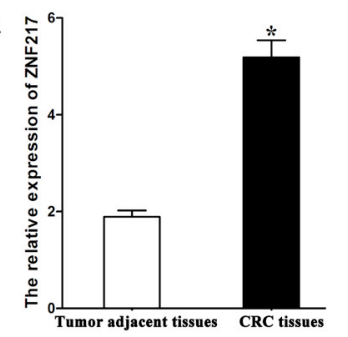

$\overrightarrow{\mathbf{B}}$
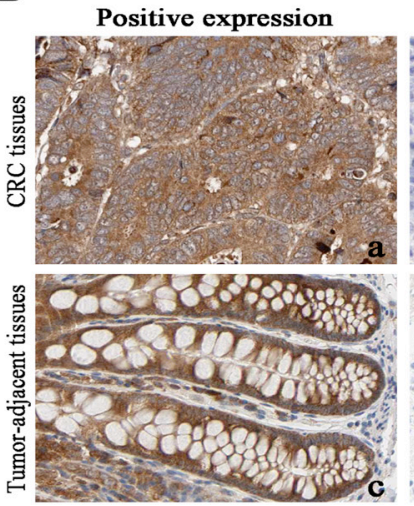

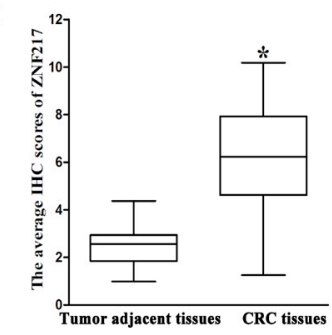

Negative expression
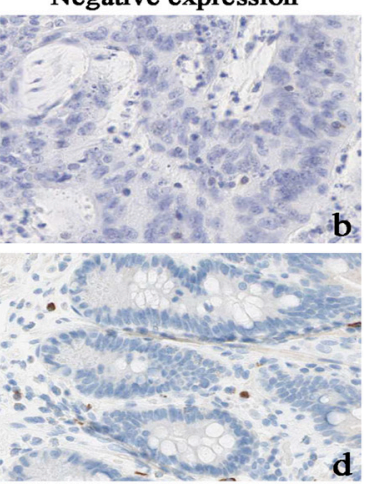

Figure 1. Different Expression of ZNF217 in CRC and Tumor Adjacent Tissues. A: The relative mRNA expression of ZNF217 was higher in CRC tissues than in tumor adjacent tissues $(5.093 \pm 0.359$ vs $1.875 \pm 0.133, * p<0.05)$. B: IHC staining in CRC and tumor adjacent tissues $(\times 400)$, a: The positive expression of ZNF217 in CRC tissues; $\mathbf{b}$ : The negative expression of ZNF217 in CRC tissues; c: The positive expression of ZNF217 in tumor adjacent tissues; $\mathbf{d}$ : The negative expression of ZNF217 in tumor adjacent tissues. C: ZNF217 obtained an epotic expression

Table 3. Clinical Correlation of ZNF217 Expression in CRC (n=60)

\begin{tabular}{|c|c|c|c|c|c|}
\hline Clinicopathologic Features & Classification & $\begin{array}{l}\text { ZNF217 Negative } \\
(\mathrm{n}=11)\end{array}$ & $\begin{array}{c}\text { ZNF217 Positive } \\
(\mathrm{n}=49)\end{array}$ & $\chi^{2}$ & $p$ \\
\hline \multirow[t]{2}{*}{ Age } & $<50$ year & 5 & 32 & 0.776 & 0.379 \\
\hline & $\geq 50$ year & 6 & 17 & & \\
\hline \multirow[t]{2}{*}{ Gender } & Male & 7 & 25 & 0.574 & 0.448 \\
\hline & Female & 4 & 24 & & \\
\hline \multirow[t]{2}{*}{ Tumor volume } & $<5 \mathrm{~cm}$ & 7 & 12 & 4.681 & $0.030 *$ \\
\hline & $\geq 5 \mathrm{~cm}$ & 4 & 37 & & \\
\hline \multirow[t]{2}{*}{ Number of nodules } & 1 & 6 & 13 & 2.092 & 0.148 \\
\hline & $\geq 2$ & 5 & 36 & & \\
\hline \multirow[t]{2}{*}{ Histopathological grade } & $\mathrm{G} 1 \sim \mathrm{G} 2$ & 8 & 21 & 3.210 & 0.073 \\
\hline & G3 & 3 & 28 & & \\
\hline \multirow[t]{2}{*}{ Lymphatic metastasis } & No & 8 & 16 & 4.457 & $0.035^{*}$ \\
\hline & Yes & 3 & 33 & & \\
\hline \multirow[t]{2}{*}{ TNM stage } & $\mathrm{I} \sim \mathrm{II}$ & 7 & 11 & 5.428 & $0.020 *$ \\
\hline & III IV & 4 & 38 & & \\
\hline
\end{tabular}



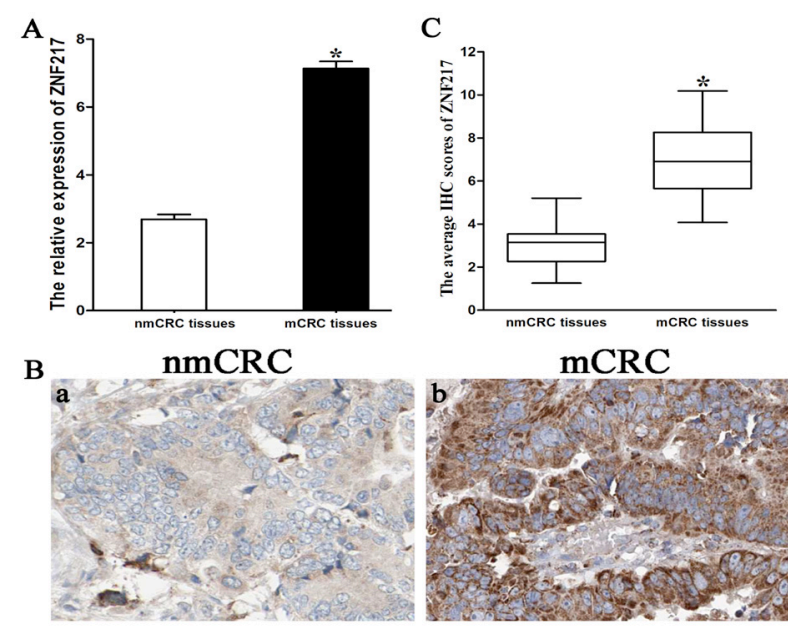

mCRC

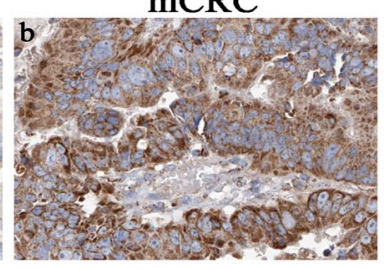

Figure 2. ZNF217 Expression in $\mathrm{mCRC}$ and $\mathbf{n m C R C}$

Tissues. A: The relative mRNA expression of ZNF217 was higher in mCRC tissues than in nmCRC tissues $(7.133 \pm 0.209$ vs $\left.2.691 \pm 0.142,{ }^{*} p<0.05\right)$. B: IHC staining in mCRC and nmCRC tissues $(\times 400)$, a: The expression of ZNF217 in nmCRC tissues; b: The expression of ZNF217 in mCRC tissues. C: ZNF217 showed an extremely epotic expression in mCRC tissues, $* p<0.05$
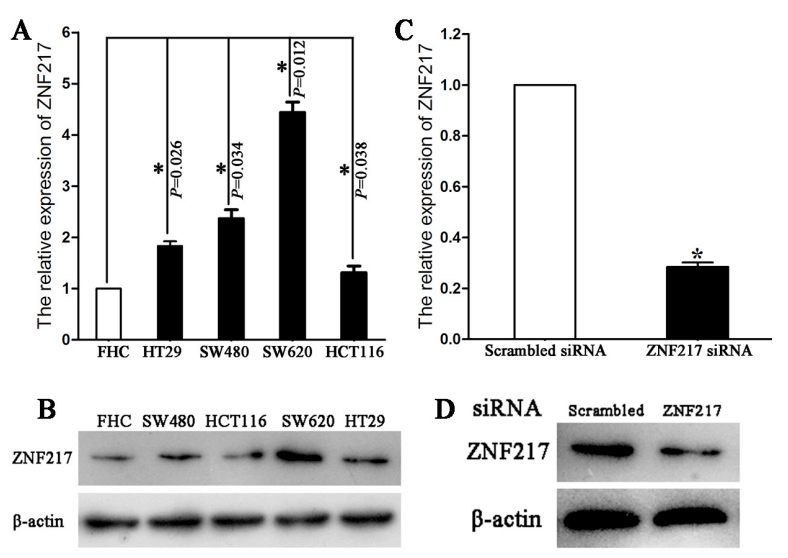

Figure 3. ZNF217 is Knocked Down by siRNA in SW620cells. A: The mRNA level in five cell lines, ${ }^{*} p<0.05$. B: The protein level in five cell lines. Data were representative of multiple repeats with similar results. C: ZNF217 mRNA expression was down-regulated in SW620 cells, ${ }^{*} p<0.05$. D: ZNF217 mRNA expression was down-regulated in SW620 cells. Data were representative of multiple repeats with similar results

can influence cell migration and invasion in SW620 cells. We designed wound healing assay to measure the changes of cell migration after knocking down the ZNF217 expression by siRNA. As shown in figure4A, we demonstrated that down-regulation of ZNF217 could significantly inhibit the relative migration distance in SW620 cells $(83.18 \pm 10.42$ vs $34.26 \pm 6.79, p<0.05$, Figure $4 B)$. In addition, we indicated that the number of invaded SW620 cells could also be impaired by knocking down ZNF217 via transwell assay $(37.45 \pm 5.38$ vs $14.73 \pm 3.17$, $p<0.05$, Figure 4C\&4D).

\section{Discussion}

Colorectal cancer is the third most commonly diagnosed cancer in the world, and it is more prevalent in developed
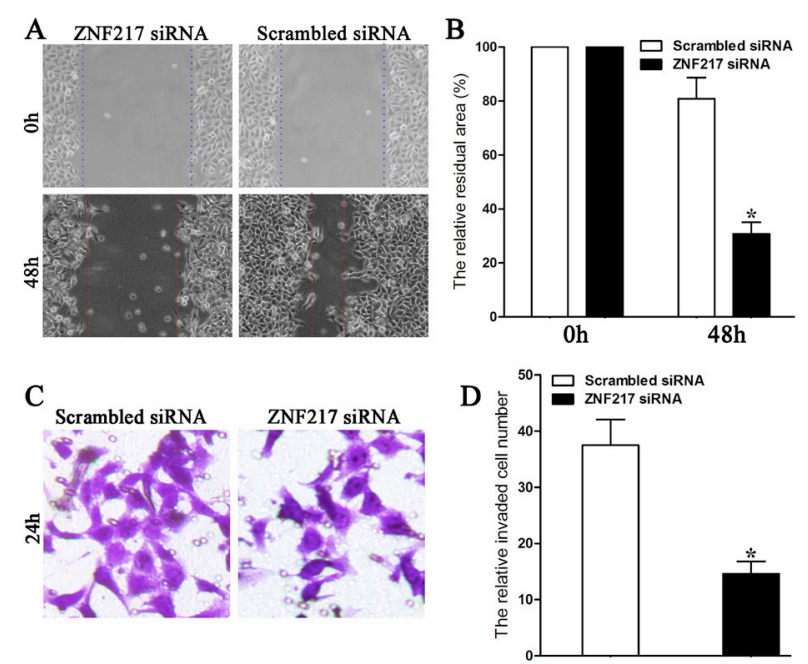

Figure 4. ZNF217 Regulates Cell Migration and Invasion in SW620 Cells. A\&B: ZNF217 knockdown inhibited cell migration in SW620 cells, 83.18 10.42 vs $34.26 \pm 6.79, * p<0.05$; C\&D: Down-regulation of ZNF217 impaired cell invasion in sw620 cells, $37.45 \pm 5.38$ vs $14.73 \pm 3.17$, $* p<0.05$

countries. According to the newest survival survey report, the CRC five-year survival rate was only $47.2 \%$ (Zeng et al., 2014). Contemporarily, radical hemicolectomy and normative chemotherapy and radiotherapy are the main therapies for CRC patients(Bozkurt, et al., 2014). However, hardly can any CRC patients be early diagnosed and easily arise lymphatic and distant metastases, consequently, identifying more effect biomarkers and therapeutic targets will become a key way to improve the survival rate of CRC patients.

Recently, in ovarian cancer, researchers found that high expression of ZNF217 could activate PI3K/Akt signal and enhance cell proliferation (Huang et al., 2014). Moreover, ZNF217 also could promote a crucial kinase, Aurora-A, for regulation of karyokinesis in breast caner cells. It could inhibit the paclitaxel-leading apoptosis which was caused by the degradation of Bcl-2 (Thollet et al., 2010). In our study, we demonstrated that the expression of ZNF217 was overexpressed in CRC tissues and associated with larger tumor volume. We may infer that ZNF217 play a tumor growth effect in CRC.

Statistical analysis certified that epotic expression of ZNF217 was positive correlated with tumor lymphatic metastasis and advanced TNM stage. In accordance with AJCC staging, both III and IV stages CRC patients lymphatic and distant metastasis, therefore, ZNF217 could enhance invasion and metastasis in CRC cells. We divided $60 \mathrm{CRC}$ patients into mCRC and nmCRC groups, and demonstrated that ZNF217 obtained a higher expression in metastatic CRC tissues. ZNF217 is one of the most important gene in chromosome of 20q13.2. Hidaka et al. (2000) reported that the copy number of 20q13.2 was increased in colorectal cancers with liver metastasis. These evidence reminded that high expression of ZNF217 in CRC tissues could facilitate liver metastasis. Cell migration and invasion are the micro-expression of tumor metastasis. In vitro, we proved that knockdown of ZNF217 suppressed cell migration and invasion in SW620 cells. Nowadays, 
in vivo experiments demonstrated that Triciribine could inhibit kinases, such as Akt and MAPK could impede cell growth and induce drug resistant (Littlepage et al., 2012). Epithelial-mesenchymal transition (EMT) is an important mechanism for tumor metastasis, and it often appear a lack of E-cadherin expression in tumor cells (Wang et al., 2013). ZNF217 could impair the expression of E-cadherin during its mRNA transcription, then induce cell occurs EMT,and eventually, enhance the ability of tumor metastasis (Bai et al., 2014).

In conclusion, the expression of ZNF217 is significantly higher in colorectal carcinoma tissues than in tumor adjacent tissues, and the up-regulation of ZNF217 is associated with malignant clinicopathological features. ZNF217 may promote CRC progression by inducing cell migration and invasion.

\section{Acknowledgements}

We thank all the patients enrolled in this study for their kind understanding and support.

\section{References}

Bai WD, Ye XM, Zhang MY, Zhu HY, et al (2014). MiR-200c suppresses TGF- $\beta$ signaling and counteracts trastuzumab resistance and metastasis by targeting ZNF217 and ZEB1 in breast cancer. Int $J$ Cancer, 6, 1356-68.

Bozkurt O, Inanc M, Turkmen E, et al (2014). Clinicopathological characteristics and prognosis of patients according to recurrence time after curative resection for colorectal cancer. Asian Pac J Cancer Prev, 21, 9277-81.

Frietze S, O'Geen H, Littlepage LE, et al (2014). Global analysis of ZNF217 chromatin occupancy in the breast cancer cell genome reveals an association with ER alpha. $B M C$ Genomics, 15, 520.

Geppert CI, Rummele P, Sarbia M, et al (2014). Multi-colour FISH in oesophageal adenocarcinoma-predictors of prognosis independent of stage and grade. Br J Cancer, 12, 2985-95.

Han K, Jin J, Maia M, et al (2014). Lower exposure and faster clearance of bevacizumab in gastric cancer and the impact of patient variables: analysis of individual data from AVAGAST phase III trial. AAPSJ, 5, 1056-63.

Hidaka S, Yasutake T, Takeshita H, et al (2000). Differences in 20q13.2 copy number between colorectal cancers with and without liver metastasis. Clin Cancer Res, 7, 2712-7.

Holzmann K, Kohlhammer H, Schwaenen C, et al (2004). Genomic DNA-chip hybridization reveals a higher incidence of genomic amplifications in pancreatic cancer than conventional comparative genomic hybridization and leads to the identification of novel candidate genes. Cancer Res, 13, 4428-33.

Huang HN, Lin MC, Huang WC, et al (2014). Loss of ARID1A expression and its relationship with PI3K-Akt pathway alterations and ZNF217 amplification in ovarian clear cell carcinoma. Mod Pathol, 7, 983-90.

Kumaravel TS, Bristow RG (2005). Detection of genetic instability at HER-2/neu and p53 loci in breast cancer cells sing Comet-FISH. Breast Cancer Res Treat, 1, 89-93.

Letessier A, Sircoulomb F, Ginestier C, et al (2006). Frequency, prognostic impact, and subtype association of $8 \mathrm{p} 12,8 \mathrm{q} 24$, $11 \mathrm{q} 13,12 \mathrm{p} 13,17 \mathrm{q} 12$, and $20 \mathrm{q} 13$ amplifications in breast cancers. BMC Cancer, 6, 245.

Li C, Yang W, Zhang J, et al (2014). SREBP-1 has a prognostic role and contributes to invasion and metastasis in human hepatocellular carcinoma. Int J Mol Sci, 5, 7124-38.

Littlepage LE, Adler AS, Kouros-Mehr H, et al (2012). The transcription factor ZNF217 is a prognostic biomarker and therapeutic target during breast cancer progression. Cancer Discov, 7, 638-51.

Mahmoud AS, Umair A, Azzeghaiby SN, et al (2014). Expression of cyclooxygenase-2 (COX-2) in colorectal adenocarcinoma: an immunohistochemical and histopathological study. Asian Pac J Cancer Prev, 16, 6787-90.

Mao XG, Yan M, Xue XY, et al (2011). Overexpression of ZNF217 in glioblastoma contributes to the maintenance of glioma stem cells regulated by hypoxia-inducible factors. Lab Invest, 7, 1068-78.

Rahman MT, Nakayama K, Rahman M, et al (2012). Prognostic and therapeutic impact of the chromosome 20q13.2 ZNF217 locus amplification in ovarian clear cell carcinoma. Cancer, 11, 2846-57.

Thillainadesan G, Chitilian JM, Isovic M, et al (2012). TGF$\beta$-dependent active demethylation and expression of the p15ink4b tumor suppressor are impaired by the ZNF217/ CoREST complex. Mol Cell, 5, 636-49.

Thollet A, Vendrell JA, Payen L, et al (2010). ZNF217 confers resistance to the pro-apoptotic signals of paclitaxel and aberrant expression ofAurora-A in breast cancer cells. $\mathrm{Mol}$ Cancer, 9, 291.

Vendrell JA, Thollet A, Nguyen NT, et al (2012). ZNF217 is a marker of poor prognosis in breast cancer that drives epithelial-mesenchymal transition and invasion. Cancer Res, 14, 3593-606.

Wang WS, Yu SL, Yang XS, et al (2013). Expression and significance of twist and E-cadherin in ovarian cancer tissues. Asian Pac J Cancer Prev, 2, 669-72.

Zeng H, Zheng R, Guo Y, et al (2014). Cancer survival in China, 2003-2005: A population-based study. Int J Cancer. [Epub ahead of print].

Zhang X, Xu J, Liu H, et al (2014). Predictive biomarkers for the efficacy of cetuximab combined with cisplatin and capecitabine in advanced gastric or esophagogastric junction adenocarcinoma: a prospective multicenter phase 2 trial. Med Oncol, 10, 226. 